\title{
ACUTE OCULAR ONCHOCERCIASIS AND ITS TREATMENT*
}

\author{
BY \\ F. C. RODGER \\ Director, West African Ophthalmic Survey $\dagger$
}

THERE are some discrepancies and a little ambiguity in the various descriptions of the clinical signs and symptoms and the course of ocular onchocerciasis. In this paper it is intended, in the light of our experiences in West Africa, to describe those manifestations of the disease in the eye which we have seen in an acute phase, and which if untreated progress in most instances to blindness. This type of blinding lesion at its simplest is limited to the cornea and the iris; adjacent tissues may, of course, be primarily or secondarily involved. Wholly on clinical grounds, we categorize the lesions as a limbitis, a sclerosing keratitis (due to the formation of pannus onchocercosus), an anterior uveitis (iritis, iridocyclitis), and a posterior exudative uveitis.

Before describing these conditions in detail, it would be as well to mention some features normally present in the African eye which may confuse the issue. Pigmentation of the eye being dense, it is not surprising that any disorder such as chronic irritation will produce aggregations of pigment and a local increase in its density. We are thinking here of irritation due to the climate and to the presence of hot winds with a high dust particle. There seems little doubt that this leads to an increase in pigmentation of the interpalpebral conjunctiva, and it is also probably the cause of the extremely frequent occurrence of pingueculae. These pingueculae show histologically (as we discovered) a well-defined hyaline plaque lying in chronically infiltrated fibrous tissue, surrounded in some instances by squamous epithelium, and within which there are several groups of fragmented, degenerate, swollen, coiled, collagen fibres. This being the characteristic histological picture of a pinguecula, there is no need for us to hypothesize that invasion of the subconjunctival space by microfilariae plays any part in the pathogenesis. The same argument holds good for early pterygia, which are also not infrequently found, although invasion of the cornea by this benign tumour is not nearly so common as in the Middle or Far East. We did notice, and have observed elsewhere (Rodger, 1957a), that the association of pigmentation of the lower bulbar conjunctiva with folds in that membrane is a more frequent finding in vitamin-A deficient areas than in areas with a plentiful supply of this essential vitamin. An interesting condition, also mentioned before (Rodger, 1957a), and called arcus tropicalis, may have the same

* Received for publication February 11, 1957.

$\uparrow$ Sponsored by the British Empire Society for the Blind. 544 
origin. In this condition, the arcus differs from arcus senilis in that there is no clear zone of cornea between the opacification and the limbus, and that its distribution is entirely superficial. The limbal pigment fuses with it.

There are one or two other pigmentary differences between the eye of an African and that of a European. Chromatophores are present in greater quantity around the perforating arteries, giving rise to an interrupted arc of dense pigment spots on the upper bulb (Fig. 1). The entire limbus is usually heavily pigmented with frond-like aggregations around the capillaries, and with the slit lamp an invasion of the cornea by pigment granules may be seen. It was noticeable that corneal neovascularization from the limbus is absent in the African eye, despite the fact that the average intake of riboflavin is only about 75 per cent. of the international requirement. Spots of pigment granules were also seen on numerous occasions passing for several millimetres over the surface of the cornea. Whether or not this migration of pigment cells is due to minor abrasion of the epithelium, we cannot say, but it seems to have no particular significance. In a very few cases, a sparse but uniform distribution of pigment granules over the entire surface of the cornea was seen in eyes that were apparently quite healthy. In the anterior uvea we observed several times an atavistic pupillary fringe, especially at the upper arc, which might be confused with old inflammatory efflorescences. In the iris stroma, it is almost the rule in the African eye for there to be a deficiency of pigment at the bottom of the crypts, and at the foot of many of the furrows. These anomalies have been selected, because here and there in the literature they have been attributed to onchocerciasis. There is no doubt that, at times-for example, when areas of depigmentation are seen

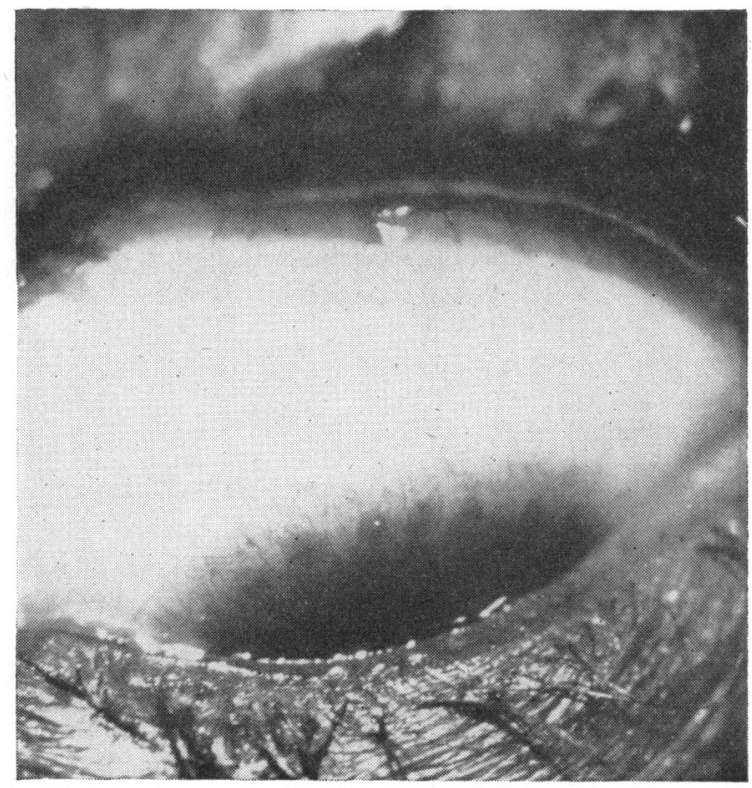

FIG. 1.-Normal Negroid pigmentation at the limbus spreads sometimes as much as $10 \mathrm{~mm}$. from the cornea, as here, fading off into the white sclera. The pigment around the perforating anterior ciliary arteries is dense and circumscribed as shown. 
in the iris stroma in association with other obvious signs of an onchocercal anterior uveitis-it is difficult to reach a decision.

\section{Onchocercal Limbitis}

This condition is seldom severe; it is characterized by discomfort more often than by pain, but it can be acute, and usually is so when a keratitis supervenes. It is due to the presence of microfilariae in the subconjunctival space, and to the death of some of them at the limbal cul-de-sac. Sometimes the patient complained of formication of the conjunctiva, but as this information was only able to be elicited by direct questioning, there must be some doubt about it. The patient suffers from lacrimation and photophobia. The clinical appearance is often very like that of a virus keratoconjunctivitis, inasmuch as there is dusky hyperaemia of the bulbar conjunctiva, a watery secretion, discomfort, and little else. However, examination with the slit lamp shows a degree of chemosis at the limbus, and in the adjacent region of the cornea a characteristic opacification which consists of a number of discrete grey dots (Fig. 2). We have described this in our notes as a "snowstorm" zone, a term which will be of use later; a few loops sprouted from the limbal vessels may be seen lying within this opacification; they pass above Bowman's membrane. We have never observed a limbitis of this type in the upper half of the cornea; it always occurs in the lower half, and may or may not involve a considerable portion of it. The commonest site of all is in the region of an inner or outer canthal pinguecula or early pterygium. The limbitis may advance to $a *$ definite sclerosing keratitis, or it may become quiescent. In the latter event it can either resolve completely, or leave behind some diagnostic evidence in the shape of a small residual sector of faint corneal opacification, frequently pigmented.

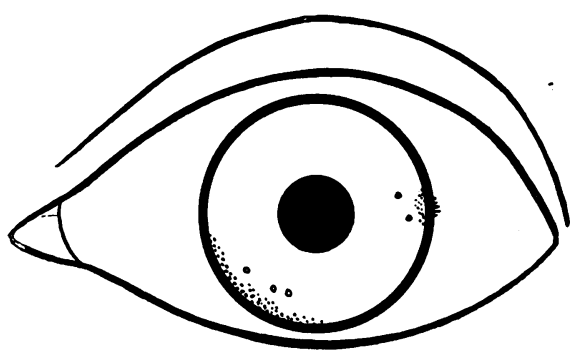

FIG. 2.-Onchocercal limbitis with an early tongue of pannus onchocercosus at 3 o'clock. The limbus at 7 o'clock is red and chemotic; in the cornea at this site there is a crescent of grey opacification (the snowstorm effect), and there are also a few punctate corneal opacities.

\section{Onchocercal Sclerosing Keratitis}

With the appearance of pannus onchocercosus, the condition becomes acute for a while at least. The keratitis frequently commences at 3 or 9 o'clock where tongues of the pannus onchocercosus invade the cornea (Figs 3 and 4); in the end they may meet at the centre (Fig. 5). Another common appearance of the sclerosing keratitis is as an apron of pannus around the lower half of the cornea (Fig. 6); this lesion also advances towards the pupillary area. Several tongues of pannus can appear at the same time in the lower half of the cornea, all of them maybe becoming confluent in the end. 


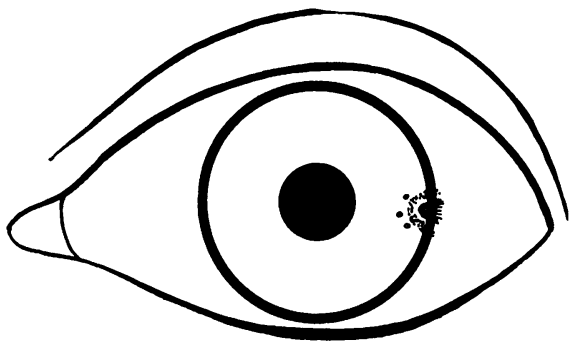

Fig. 3.-Early stage of onchocercal sclerosing keratitis, originating at a pinguecula. Three zones can be seen: an advancing "snowstorm" edge, a dense white middle zone, and a pigmented basal zone. A few punctate opacities are related closely to the advancing edge.

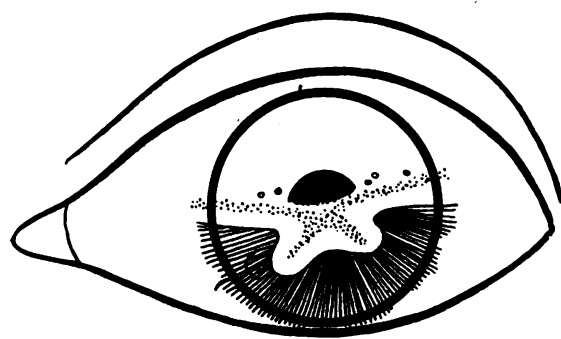

Fig. 5.-An advanced case of onchocercal sclerosing keratitis formed by the fusion of three separate tongues of pannus. The characteristic zones comprising each tongue are shown here, but the picture is often less clear-cut.

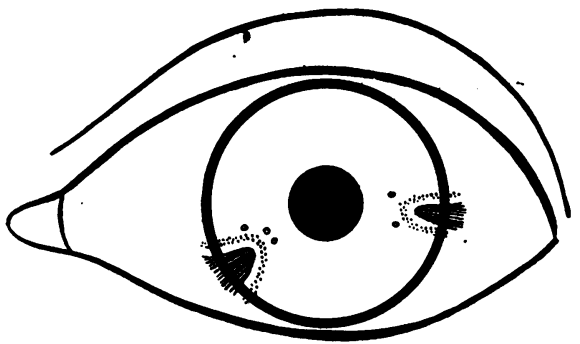

Fig. 4.-Two tongues of pannus onchocercosus.

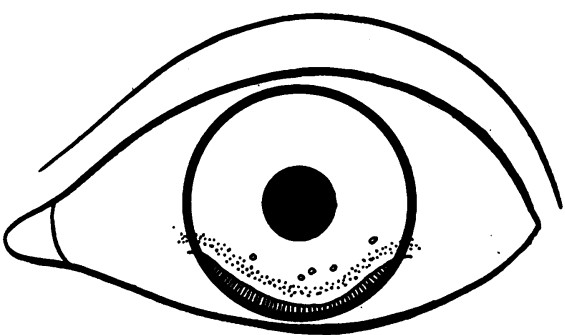

Fig. 6.-An apron of pannus onchocercosus, which has developed from a limbitis. Although punctate opacities related to pannus have been drawn in each instance, they are not always present.

When the condition is allowed to advance unchecked, the end-result is total involvement of the cornea; on many occasions we have seen such a pannus advance until all that was left was a clear area at 12 o'clock (Fig. 7); this is quite a common finding.

FIG. 7.-The end-result of onchocerciasis of the cornea. Large vessels have appeared superficially in the pigmented basal zone. A view of the iris can often be obtained through the comparatively clear zone at 12 o'clock.

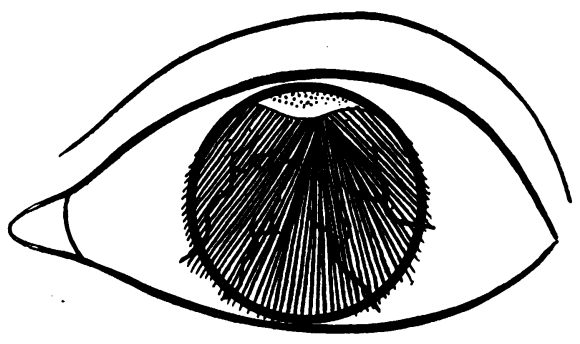

The external appearance of the pannus onchocercosus is fairly characteristic: it lies at first between the epithelium and Bowman's membrane, as would a pannus degenerativus, but differs from the latter in being vascularized. Subsequently, it may break through Bowman's membrane or appear well below it. It is often associated (because the cornea is invaded in force) with interstitial areas of fibrosis. 
The pannus consists of three zones: an advancing "snowstorm" zone of separate grey dots, a middle, white, zone, where the individual opacifications are densely packed, and a basal pigmented zone, which well illustrates the propensity in the African eye of the pigment cells to follow in the path of any traumatic or inflammatory process (Fig. 8). In the end, the epithelial surface above the pannus may become entirely pigmented, although it can be seen under high magnification to fade off towards the upper pole of the cornea. Corneal neovascularization is essentially restricted to the pannus, although in late cases, as indicated, new bloodvessels may break through into the stroma.

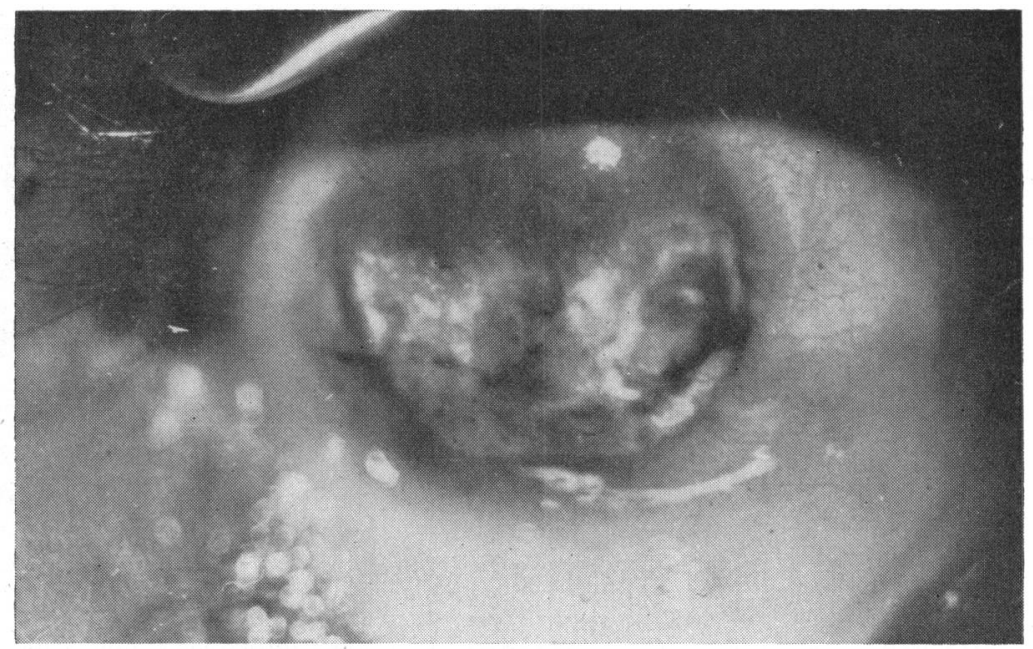

Fig. 8.-Advanced stage in the sclerosing keratitis of onchocerciasis. The three basic zones of pannus onchocercosus may be made out. There are some punctate opacities associated with the advancing edge of the pannus.

At all stages in this condition, one finds evidence of corneal degenerations. The keratitis is quite frequently associated with an anterior uveitis, and it appears from our records that degenerations of the corneal epithelium occur more frequently when such an association exists. Epithelial cysts and calcification may be seen; a band-shaped opacity is a fairly frequent finding in an early onchocercal sclerosing keratitis, even when the anterior uvea is not affected. Xerosis corneae is another common complication. One assumes that the major factor in all this is interference with corneal diffusion as a result of involvement of the limbal circulation.

An onchocercal punctate keratitis has been described as a separate entity, quite distinct from the sclerosing keratitis described here. In another paper (Rodger, 1957b), this lesion has been fully discussed in relation to the other types of punctate keratitis in Africa. There is no doubt that, in association with a limbitis or with tongues of pannus onchocercosus, punctate-like opacities are often seen. We should like to stress, however, before leaving this question, that a punctate keratitis in a quiet eye without concomitant signs of onchocercal keratitis or limbitis does not in our view occur. The diagnosis of an onchocercal keratitis does not, in short, depend upon punctate corneal opacities, an opinion also held by Hissette (1932). 


\section{Onchocercal Anterior Uveitis}

As already mentioned, this condition can occur either by itself or in association with a sclerosing keratitis. It can be an extremely acute lesion subject to recrudescences. On the other hand, it often appears to remain sub-acute or chronic. We should like to describe-for it does not seem to have been described beforethe acute manifestation.

The symptoms are those of an acute granulomatous iridocyclitis due to any cause. The iris is invaded, as we have proved to our own satisfaction by performing biopsies on several such cases, by living microfilariae, on the death of which gross exudation occurs at the pupillary margin. This is an important and specific sign of the condition. The exudation very quickly completely fills over the entire pupil. At the same time, the aqueous changes in nature and flare can be seen. Keratic precipitates are not a feature of the condition, but a few are sometimes seen in the centre rather than in the lower half of the cornea. The exudation over the pupil can increase in size until it either becomes fixed to the posterior surface of the cornea or spills over into the angle of the eye at 6 o'clock. As a result of this attachment, the pupil is characteristically deformed in that direction. Not surprisingly, as the greatest site of the inflammation is at the pupillary margin, the posterior pigmented fringe may become attached to the anterior lens capsule. In this, the characteristic lesion, the condition is strictly an iritis; however, very often the inflammatory process involves the ciliary body as well. As the entire picture occurs as a result of the disintegration of dead microfilarial bodies, it is invariably self-limiting. Recurrences, however, are common. After a period of a few days, even if untreated, the lesion usually begins to quieten down, adopting a subacute or chronic course. Unfortunately, the exudate often does not resolve to any extent at all; in short a very common end-picture is that of total occlusion of the pupil (Fig. 9). In several cases observed, where resolution did occur, it

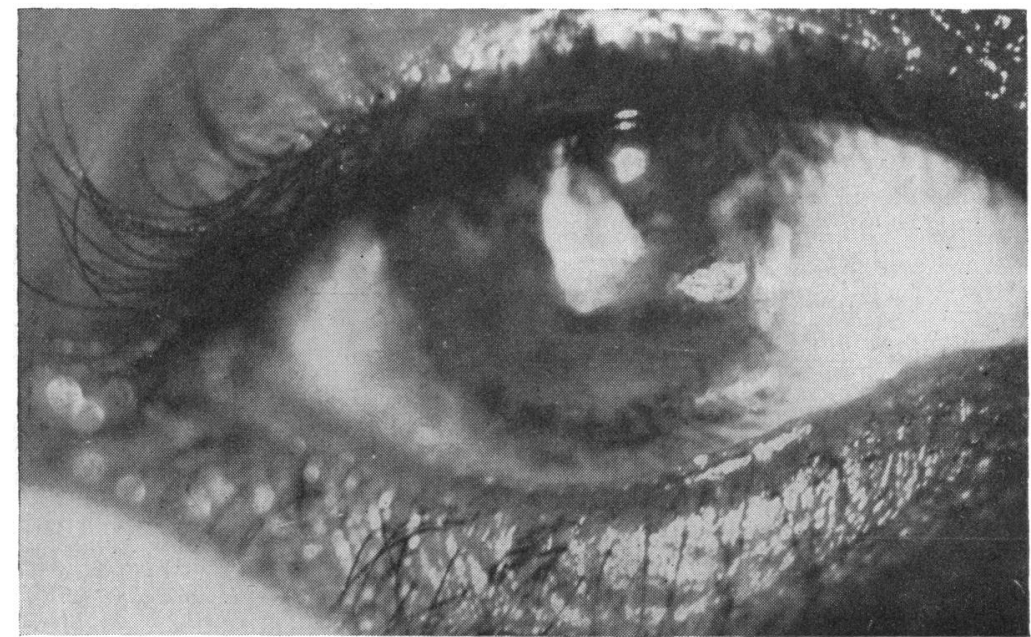

Fig. 9.-End-result of onchocercal anterior uveitis. The pupil is occluded by a fibrous membrane, and is deformed by two anterior synechiae, the lower of which can be seen attached to the cornea at 3 o'clock in the neighbourhood of a tongue of pannus onchocercosus. Pigment invasion of the cornea below is the site of an old limbitis. The middle and basal zones of the pannus can be made out. 
appeared to take place in a characteristic way; as a result of shrinkage, the pupillary membrane splits and patent sectors appear, usually at the pupillary margin. If the acute phase does not last long, then the lens behind is unlikely to be affected, and the patient will retain some measure of vision through these apertures in the inflammatory membrane. We have seen many cases where the entire exudative membrane disappeared, leaving in its wake a "rolled" border of organized exudate completely surrounding the pupil; in other cases not even this remained, the only evidence being the total depigmentation of the posterior pigmented fringe. The partial absorption and shrinkage of the anterior chamber mass (when it exists), which, as we have shown, usually remains attached to the pupillary margin, results in further distortion of the pupil in a downward direction. A not infrequent finding was that of a piece of organized exudate somewhere at the root of the iris whence it had fallen during the course of the acute phase forming an anterior synechia. Residual posterior synechiae with associated patches of iris atrophy were quite common, as was smearing of the entire pigment fringe over the anterior lens capsule. Pigment particles, fairly large in size, detached from the posterior pigment fringe, were quite often seen adherent to the remnants of the exudative membrane, or even to the synechiae. In many cases, the iris furrows become obliterated with pigment, the final appearance being that of brown velvet. The posterior attachments are sometimes associated with spots of opacification in the lens, which are sub-capsular. The characteristic miscellany of colours displayed in a posterior complicated cataract were also sometimes seen in association with precipitates on the posterior lens capsule, thereby furnishing further evidence that during the acute attack the ciliary body may be involved. The commonest type of complicated cataract was one where the anterior capsule had become white and thick, with exudative remnants attached, being separated from the shrunken lens body by white sterile fluid. It is tragic to think that often in such an eye the only residual defect preventing the subject from seeing is an inoperable complicated cataract. When the lens and ciliary body are involved, contracture of the cyclitic membrane leads to degeneration of the suspensory ligament, the lens frequently becoming subluxated or completely dislocated.

We never saw ectasias in association with onchocercal uveitis; nevertheless it is true to say that acute inflammation of the sclera is commonly associated with the sclerosing keratitis, the anterior uveitis, and the posterior uveitis now to be described. As a result of broad anterior synechiae at the periphery, secondary glaucoma is common.

\section{Onchocercal Posterior Exudative Uveitis}

The descriptions of the posterior segmental lesion of onchocerciasis are so varied as to underline the fact that this lesion is the least understood. Through the many different descriptions one alone appears to be specific, and that is essentially a choroidal sclerosis involving the periphery either before or at the same time as it destroys the macula. This lesion is slow in onset and is never acute. It will be discussed in another paper. One reference only to this interesting condition will be made, and that is that the older view as to its aetiology is now out of favour; many more cases of the posterior segmental lesion have been found in association with low degrees of infection than with high, and in pathological specimens more eyeballs exhibiting this lesion have been found without microfilariae in the choroid 
than with them. If the typical posterior segmental lesion of onchocerciasis does not necessarily depend, then, upon the presence of microfilariae in the tissues involved, what type of lesion does arise as a result of their death in the posterior uvea? We believe this is what happens:

In seven of 27 cases of acute anterior uveitis there was a concomitant posterior exudative uveitis. In four of these cases we were fortunate enough to enucleate the eyes. We were, therefore, able to confirm the presence of microfilariae in the posterior segment. Owing to the anterior involvement it was not easy, except in a few cases, to observe the fundus, and so the clinical description of this condition which we shall give now may not be as accurate as it should be. The retinal pigment epithelium reacted to the presence of the parasites (or their dead bodies) by the double process of degeneration and proliferation; this occurred more grossly than in the degenerative type of the posterior lesion. Large focal masses of pigment were scattered here and there, and occasional glimpses of the choroidal vascular system were observed; these vessels were usually healthy if somewhat engorged. In a few cases, small retinal haemorrhages were seen at the posterior polar region. Here and there, white patches indicated areas of choroido-retinal fusion, usually with retinal pigment heaped up at the edge. Exudates in the anterior chamber or even in the vitreous made it impossible to observe fuller details, but in one case the optic disc was atrophic and deeply cupped. Colloid bodies appeared to be common. They are, of course, not part of the acute picture, but were present in most of the quiescent cases of this condition that we saw. They may be taken as evidence of the previously acute inflammatory state of the uvea.

We have described briefly the appearance of the posterior uvea when that tissue is invaded by microfilariae. It is essentially part of an exogenous endophthalmitis. Recurrences must be common, and there can be no doubt this is a prolific cause of blindness. Quiescent cases with characteristic anterior lesions were extremely numerous, and at such a time the picture is that of a chronic non-specific uveoretinitis. The association with a typical anterior lesion, however, usually points to the diagnosis. In the absence of the latter, it is not possible to diagnose a non-specific posterior uveitis as due to onchocerciasis with certainty; nevertheless, it could happen.

\section{Treatment of Acute Conditions}

It is generally accepted to-day that the pathogenesis of onchocerciasis of the cornea and iris depends upon the death in these tissues of the parasites. There seems no reason to doubt this belief. At the outset of treatment, therefore, one is faced with this problem: if a drug is administered which kills the microfilariae, might this not exacerbate the symptoms and further increase the visual defect by increasing the number of dead microfilariae in the ocular tissues? A few such cases have been reported in the literature. For this reason we approached the problem very carefully.

To begin with, using diethylcarbamazine, we treated our cases of acute onchocercal kerato-uveitis with a standard course of 200 tablets, that is $10 \mathrm{~g}$. Despite our fear that the ocular condition would worsen as a result of this treatment, there was no doubt that after 6 or 7 days it settled; on the other hand, there was little evidence of recovery of sight. In short, all that was achieved was a quiet, painless, blind eye. With the slit lamp we observed 
that there was some degree of resolution of the keratitis, especially in the case of any punctate opacities lying close to the pannus, although little recovery at the pupil occurred.

Dissatisfied with our results, we turned to non-specific protein therapy, and here from the very first the effect was striking. We administered TAB vaccine intravenously, and also tried autohaemotherapy. The former was the more successful especially when we gave large doses. We started with 5 million units; a second dose of 10 million units was given, and finally one of 15 million units. As the effect of this therapy was immediate after the first injection, it was soon found best to standardize the treatment by administering a single dose of 10 million units of TAB vaccine, and following it up, as soon as the temperature had come down to normal, with the filaricide (10 g. Hetrazan). The effect of the protein therapy was to quieten the eye in a dramatic way. This was particularly true of the uveitis. The eye became quiet, and lacrimation and photophobia disappeared. A great deal of the ciliary and conjunctival hyperaemia vanished, and the cornea regained much of its lustre. When the filaricidal course was started, there was a reappearance of the acute symptoms, but it did not appear to be so distressing as when this drug was given without the initial protein therapy. Of course, control of this last finding is quite impossible, and the claim may not in fact be true. However, the ultimate result when protein therapy preceded filaricidal therapy appeared to be much better. Resolution of the occluding membrane was expedited, and return to a quiet eye occurred

\begin{tabular}{|c|c|c|c|c|}
\hline $\begin{array}{c}\text { Serial } \\
\text { No. }\end{array}$ & $\begin{array}{l}\text { Age } \\
\text { (yrs) }\end{array}$ & I.D.F.* & Lesion & Protein Therapy $\overrightarrow{\overline{0}}$ \\
\hline 15 & 35 & 31 & Acute kerato-uveitis & $10 \mathrm{ml}$. own blood intramusca \\
\hline 221 & 46 & 31 & Acute kerato-uveitis & Nil \\
\hline 595 & 35 & 31 & Acute anterior uveitis & $\begin{array}{l}20 \text { ml. own blood intramuscu } \\
10 \text { million units T.A.B. intrave } \\
\text { then given }\end{array}$ \\
\hline 606 & 20 & 31 & Acute keratitis . & $\begin{array}{l}5 \text { million units, no effect; } 10 \\
\text { disappointing; } 15 \text { millio } \\
\text { T.A.B. intravenously }\end{array}$ \\
\hline 865 & 23 & 31 & Acute kerato-uveitis & $\begin{array}{l}2.5 \text { per cent. Cortone topicas } \\
\text { through the day; } 25 \mathrm{mg} \text {. d } \\
\text { cortone daily for } 7 \text { days } \\
10 \text { million units T.A.B. intray }\end{array}$ \\
\hline
\end{tabular}


sooner. The chance of preventing permanent total blindness was increased. Where cases have been reported in the literature of a slight improvement in the visual acuity after the excision of nodules, the modus operandi may well be that of a non-specific protein therapy resulting from the trauma of the surgical procedures.

The results of five of the more interesting cases in the clinical trials with this combined therapy are given in the Table.

Antihistamine and cortisone proved of little if any value. The depressing effect of the former is in itself a contraindication. We soon abandoned it altogether. Atropine we prefer to use after the TAB has quietened the eye, as many of the acute cases show a rise in tension.

\section{Discussion}

The lesions we have described are acute, and as such are not often seen. There is no doubt that in an out-patient clinic in a hospital in Africa they are extremely rare. In the course of our survey in Ghana (which was the most heavily infected region we visited) we noted 59 acute cases (excluding cases of limbitis). The disagreement in the literature whether or not ocular onchocerciasis ever has a really acute phase in the full meaning of the word will bear some reference now.

Hissette (1932) stated that, in many of his cases, although conjunctival hyperaemia and a ciliary flush were present, the iritis was very mild indeed. He said this was the characteristic picture, calling it a "torpid" iritis.

PF ACUTE ONCHOCERCIASIS

\begin{tabular}{|c|c|c|c|}
\hline \multirow{2}{*}{ Result } & \multirow{2}{*}{ Effect of Hetrazan } & \multicolumn{2}{|c|}{ Visual Acuity } \\
\hline & & Before & After \\
\hline \multirow[t]{2}{*}{ e } & Photophobia and lacrimation for 5 days & C.F. & $6 / 60$ \\
\hline & $\begin{array}{l}\text { Gross oedema of lids and cheek } \\
\text { Photophobia } \\
\text { Blepharospasm } \\
\text { Lacrimation for } 7 \text { days }\end{array}$ & H.M. & C.F. \\
\hline uiet now & $\begin{array}{l}\text { Acute flare-up after one tablet; course interrupted } \\
\text { Nil }\end{array}$ & C.F. & $6 / 36$ \\
\hline e & Slight lacrimation only & C.F. & $6 / 36$ \\
\hline$t$ & & & \\
\hline e & Lacrimation and photophobia for 2 days & $\begin{array}{l}\text { R6/12 } \\
\text { L6/18 }\end{array}$ & $\begin{array}{l}6 / 12 \\
6 / 9\end{array}$ \\
\hline
\end{tabular}

d Brown, 1956) 
Strong, Sandground, Bequaert, and Ochoa (1934), who studied this condition in Guatemala in 1930, and later visited Hissette in the Congo, state that in Central America the ocular disturbances generally have an insidious onset. The only exception to this is the fact that photophobia was complained of in some of his cases. This picture is not one of a really acute condition.

Ridley (1945) is of the opinion that a chronic conjunctivitis with some acute exacerbations is the first ocular complaint, to which is added a degree of photophobia when corneal complications begin. Lesions of the anterior segment of the eye, he says, are usually insidious, giving rise to a slow but steady deterioration. In general, the finding of the more advanced ocular lesions in those of middle age shows, Ridley argues, that chronicity is the rule, and it is probable that the disease may run some 10 years before producing complete blindness.

Scott (1945) gives no indication of the clinical symptoms of onchocercal keratitis. He describes the iridocyclitis as developing with an accompanying photophobia, lacrimation, and ciliary flush, but holds to the published view.

Toulant and Boithias (1952), discussing the functional troubles which keratitis provokes, say they would be "less marked or less easily appreciated in the eyes of Negroes than they would be in the eyes of Europeans". This remark is not without some significance. They also note that, although they have seen the insidious iritis described by Hissette and the rest, they have also seen a plastic iritis with synechiae which must at one time have been acute.

This rapid survey of the literature shows the general consensus of opinion to be that the keratitis of onchocerciasis is a sub-acute condition, and that the anterior uveitis is an insidious one. A posterior uveitis of the type described here has not been described before. It is probably this condition which bas led to such confusion in the many and varied descriptions of the posterior degenerative lesion of onchocerciasis, which has almost certainly quite a different aetiology.

The present paper shows clearly that acute ocular onchocerciasis affecting the cornea and the uvea are found in West Africa. There may be several reasons why this has not been noted before except in the conjectured reference to it by Toulant and Boithias. It is not to be forgotten, when dealing with the whole subject of onchocerciasis, that much depends upon the density of the infection of the subjects in the endemic areas, for the degree of invasion of the ocular tissues must surely reflect the density of the infection in the individual. We have shown to our own satisfaction that the pathogenesis of these ocular lesions depends upon the death of the parasites in the tissue concerned; this is the general view, and it should be readily accepted that where one microfilaria dies there will be a much milder reaction than where a dozen die, other things being equal. This is one possible explanation of the discrepancy in the various reports of the ocular symptoms of the disease. 
We have visited areas of varying degrees of endemicity, and there appears to be some support for just such a point of view in what we found. In areas of low endemicity, the density of infection in the individual tended to be equally low and acute cases were few and far between. We have used an index to measure this density (Rodger and Brown, 1957). It was only when the Individual Density Figure (IDF) was 31 (the optimum figure), or very nearly so, that an acute eye was found. Nevertheless, the reverse is not true; many of the cases which clinically were chronic in type had equally high Individual Density Figures. In these latter cases, some other explanation must be found for the absence of acute symptoms. We believe that the answer is an immunological mechanism. This will be the subject of a further communication.

Although we have said that the intensity of the clinical symptoms depends upon either a quantitative or an immunological factor, there still must remain in territories where everybody over 20 has the disease, and where the Individual Density Figures are extremely high, many more cases of acute ocular onchocerciasis than those we find reported. The answer here, we feel, lies in the fact that the acute condition lasts for such a short time. During this period the subject has little inclination to attend a hospital. This is partly due to the inaccessibility of the villages and partly to the psychology of the people. When in pain, they hide. It is the acute case, as we have said, which is the more certain to progress to total blindness; we believe that sub-acute or chronic cases seldom do so, until an acute exacerbation occurs. Unless it is recognized that these different clinical manifestations of the disease exist, it would be quite easy to make the mistake of supposing that the course of the disease was always insidious before total blindness ensued. Although Ridley (1945) has argued that the finding of the more advanced ocular lesions in those over middle age proves that chronicity is the rule, it could be equally well argued that this finding is merely indirect evidence that acute blinding crises occur in all age groups. We believe that to be so. It greatly alters the prognosis and treatment.

As far as the clinical signs are concerned, there is on the whole fair agreement. We find it useful to distinguish between limbitis and sclerosing keratitis, because the former is not always an early stage of the latter; the condition in many cases never advances beyond the stage of a limbitis. Whether or not other workers agree with our view that a punctate keratitis is not in itself a manifestation of ocular onchocerciasis does not matter, because the condition is never a blinding one and so does not come into the scope of this paper. The proportion of blindness due to the keratitis and to the uveitis is almost equal, although taking those cases where both conditions exist, it is invariably true that it is the uveitis which leads to blindness. In a heavily endemic area in Northern Ghana, we noted 59 acute cases (excluding limbitis cases) of ocular onchocerciasis out of 450 sufferers from the disease. These 450 patients all had some defect of vision, in other 
words they were a selected sample, although the causes of blindness were not restricted to onchocerciasis. Of the 59 acute cases, 27 suffered from onchocercal anterior uveitis (seven in association with posterior inflammation), 22 from the sclerosing keratitis, and ten from a mixture of both; 42 of the 59 were blind.

It is interesting to note the distribution of age:

In patients aged 15 years and under, there were six acute eyes: three of these were blind, the cause being a uveitis in one and a keratitis in two. All the three who retained some measure of vision suffered from the keratitis.

In the age group 16-25, there were fifteen acute lesions, of which seven were blind. The cause was a uveitis in three, keratitis in one, and kerato-uveitis in three. In the eight who retained their sight, the cause was a uveitis in two, a keratitis in five, and a kerato-uveitis in one.

In the age group 26-45, there were 33 acute lesions, of which 27 were already blind. The cause was a uveitis in fifteen cases, a keratitis in seven, and a keratouveitis in five. The seven cases exhibiting a posterior uveitis were all in this group. Of the six with some degree of visual acuity left, the cause was a uveitis in two, a keratitis in three, and a kerato-uveitis in one.

Finally, in the age group oyer 45 , there were five acute cases of onchocerciasis, all of which were blind, four as a result of uveitis and one as a result of keratitis.

It is not intended, in giving the details in these groups, to make any strictly significant statistical deductions: nevertheless, it is obvious that acute lesions of ocular onchocerciasis are not uncommon in a heavily endemic area, and that they occur in all age groups. The average Individual Density Figure for those blinded by an anterior uveitis was 28 , as compared with 26 in the case of those blinded by the keratitis; there is little material difference here. In those acute cases where some degree of vision was retained, the average Individual Density Figure was 22; neither is this, in our view, significant. What is significant is that the Individual Density Figures in all these acute cases were high. In fact, where it is low one would tend to be doubtful of the diagnosis. While the sclerosing keratitis is a fairly easy picture to diagnose even at an advanced stage, many of the cases of uveitis are not. This is particularly true in patients about 50 years of age where the disease in the skin may have died out. If there is any doubt in diagnosing uveitis in an eye now quiet, allowance being made for age, we personally should not feel like accepting that diagnosis unless there were nodules present, or at least one of the eight skin-biopsy series recommended by us (Rodger and Brown, 1957) was positive; the majority of quiescent uveitis cases seen are above 45 years of age, so this is quite a problem. The proportion of cases involving one eye to those involving two is in the ratio of $1: 7$. This factor should also be of some help in formulating a diagnosis based on the clinical signs. 


\section{Summary}

(1) Acute manifestations of ocular onchocerciasis are seen as a limbitis, a sclerosing keratitis, and as an anterior and posterior uveitis.

(2) Differences in the African eye from the European eye that might confuse the clinical picture are discussed.

(3) The clinical signs and symptoms of onchocercal limbitis, sclerosing keratitis, and anterior and posterior uveitis are fully described.

(4) The lesions are usually seen in a sub-acute or chronic stage, but it is believed that in most of them there has been or will be an acute crisis at some time or other.

(5) After a few days the acute phase usually settles down, adopting once again its sub-acute or chronic course; in some instances, it is the acute manifestation which occurs first.

(6) It is during the acute attack that blindness is most likely to occur and consequently it is believed that it is most important to treat the acute cases.

(7) The treatment found to be most successful during the acute phase was an initial intravenous injection of 10 million units of TAB vaccine, followed as soon as the temperature was normal by $10 \mathrm{~g}$. diethylcarbamazine (Hetrazan) orally. The eye becomes quiet within 12 hours of the nonspecific protein therapy; although, on exposure to the filaricide, lacrimation and photophobia return, nevertheless the symptoms are not so acute as before. Resolution appears to be more likely to occur when protein therapy is administered first.

(8) A discussion of the clinical signs and symptoms in the light of the findings of other workers is given. It is noted that the general consensus of opinion is that the keratitis is a sub-acute condition and the anterior uveitis an insidious condition. The observations and results in the present paper show this not to be the case. Reasons for the discrepancies between the findings of various authors are put forward.

(9) It is suggested that the intensity of the clinical symptoms depends upon quantitative and immunological factors. The evidence of this is to be reported in a further communication.

(10) The description given of an exogenous posterior exudative uveitis due to onchocerciasis is believed to be the first publication of this finding.

\section{REFERENCES}

Hissette, J. (1932). Ann. Soc. belge Méd. trop., 12, 433.

RIDLEY, H. (1945). British Journal of Ophthalmology, Monograph Suppl. X: "Ocular Onchocerciasis". Pulman, London.

RODGER, F. C. (1957a). Amer. J. Ophthal. (In the press). (1957b). British Journal of Ophthalmology, 41, 513.

and Brown, J. A. C. (1957). Trans. roy. Soc. trop. Med. Hyg., 51, 271.

ScotT, J. G. (1945). Amer. J. Ophthal., 28, 624

Strong, R. P., SAndground, J. H., Bequaert, J. C., and OchoA, M. M. (1934). "Onchocerciasis". Harvard University Press, Cambridge, Mass.

Toulant, P., and Boithias, R. (1952). Bull. Soc. Path. exot., 45, 811. 